\title{
Variation in task complexity and adult age differences in frequency-of-occurrence judgments
}

\author{
DONALD H. KAUSLER, RUTH E. WRIGHT, and MALEKEH K. HAKAMI \\ University of Missouri, Columbia, Missouri 65211
}

\begin{abstract}
Young adult and elderly subjects were contrasted in performance on a relative frequency judgment task under intentional and incidental instructions and with and without performance on an ancillary case-monitoring task. In agreement with earlier studies, null effects were found for instructional variation and for the Age by Instructions interaction. Frequency judgment scores were adversely affected by ancillary task performance. However, the adverse effect was no greater for the elderly subjects than for the young subjects, in agreement with the hypothesis that the encoding of frequency information is insensitive to age changes. Unlike earlier studies, the young subjects were superior overall to the elderly subjects in frequency judgment accuracy. The age difference was attributed to an age-sensitive effortful retrieval process that is operative even on relative frequency judgment tasks and is related to level of fluid intelligence.
\end{abstract}

The frequency of occurrence judgment task has assumed importance in memory research largely through its apparent involvement of an automatic encoding process. The primary criterion of its automaticity is its insensitivity to variation in instructions (Hasher \& Zacks, 1979). That is, performance proficiency is no higher under intentional instructions than under incidental instructions. A further criterion of its automaticity appears to be its insensitivity to adult age variation. Null effects for age variation have been found for the relative frequency judgment task in which pairs of items are presented and subjects select the member of each pair believed to have occurred more frequently in a prior study list (Attig \& Hasher, 1980; Kausler \& Puckett, 1980).

On the other hand, modest, but statistically significant, adult age differences have been found for the absolute frequency judgment task in which individual items are presented and subjects estimate the number of times each item had occurred in a prior study list (e.g., Warren \& Mitchell, 1980). The age difference for absolute judgment scores does not seem to be attributable to a more conservative response criterion established by elderly adults than by young adults (see Warren \& Mitchell, 1980). Automatic memory encoding processes, as defined by insensitivity to instructional variation, may, like effortful encoding processes, exhibit modest deficits in proficiency from early to late adulthood. The absence of adult age differences on the relative judgment task may simply be a manifestation of

Requests for reprints should be addressed to Donald $\mathrm{H}$. Kausler, Department of Psychology, 210 McAlester Hall, University of Missouri, Columbia, Missouri 65211. This research was supported by NIA-USPH Grant 1 R01AG01485-02, awarded to the first author. a ceiling effect. Scores are ordinarily quite high for this task, sufficiently high to mask what could be the superior encoding proficiency of young adults. Alternatively, the greater involvement of an age-sensitive retrieval process (Craik, 1977) on the absolute than on the relative judgment task may account for the presence of adult age deficits on the former task only.

The present study attempted a more rigorous test of the hypothesis that the encoding of frequency information is insensitive to a decrement in proficiency over the adult life span than the tests conducted in earlier studies involving relative judgments. The overall strategy called for introducing a condition in which the encoding of occurrence information is interfered with to some degree. If the encoding of occurrence information is an age-sensitive process, then the interference should be greater for elderly adults than for young adults. Whatever age deficit is present under a standard condition should be magnified greatly under an interference condition. Half of the present study list items were printed in uppercase letters, and half were presented in lowercase. Subjects in the interference condition performed an ancillary monitoring task in which they decided whether or not the case format of an exposed item matched that of the previously exposed item. Subjects in the standard condition simply studied the items without performing the ancillary task.

The reason for the expected interference rests in the nature of the encoded information mediating frequency judgments. The prevailing conceptualization of the frequency judgment task is provided by Hintzman's multiple-trace theory (e.g., Hintzman \& Block, 1971). According to this theory, each perception of an item yields a separate trace of that item's sensory and/or semantic features. Retrieval of the separate traces offers sufficient information to evaluate, either absolutely or 
relatively, the frequencies of occurrence of the individual list items. Since an item's case format is shared by many other items in the study list, its processing could not provide distinctive features for later use in estimating that item's number of occurrences. Processing case information should, therefore, interfere with the processing of an item's distinctive sensory/semantic features, the kind of processing that is essential for establishing separate retrievable traces of that item. Consequently, performance proficiency on even a relative frequency judgment task should decrease, relative to proficiency under the standard condition.

\section{METHOD}

\section{Subjects}

The 48 young adult subjects ( 33 women, 15 men; mean age $=18.6$ years, range $=17-27$ years $)$ were undergraduate students, and the 48 elderly subjects ( 35 women, 13 men; mean age $=70.1$ years, range $=55-86$ years) were residents of Columbia, Missouri. The mean and standard deviation for years of formal education were 13.20 and .47 years for the young subjects and 15.62 and 2.93 years for the elderly subjects $[t(94)=6.03, p<.001]$. Following the frequency judgment task, each subject was administered the Figural Relations Test of Horn's Gf-Gc sampler as a means of assessing level of fluid intelligence. ${ }^{1}$ Scores on this test have been found to correlate moderately with relative frequency judgment scores for young adults, but not for elderly adults (Kausler \& Puckett, 1980). All 48 young subjects completed the test, with a mean and standard deviation of 10.52 and 2.64 years, respectively. Only 33 of the elderly subjects completed the test, with a mean and standard deviation of 6.30 and 3.20 years, respectively. The age difference in means was significant $[\mathrm{t}(79)=6.12, \mathrm{p}<.001]$.

\section{Design}

The design was a 2 by 2 by 2 by 6 mixed factorial in which the first three variables were between-subjects and the fourth was within subjects. Between-subjects variables were age variation (young vs. elderly adult), ancillary task variation (presence vs. absence of case monitoring), and instructional variation (intentional vs. incidental). The within-subjects variable consisted of pair type in the test phase $(0: 1,0: 3,0: 5,1: 3,1: 5$, and $3: 5$; the numbers refer to the frequencies with which test pair members had occurred in the prior study list). Mean age, educational level, and Figural Relations Test scores were all roughly comparable for the four conditions at each age level.

\section{Materials}

The 81 -item study was identical in item content to one of the two lists employed by Kausler and Puckett (1980) (the two lists in the earlier study were comparable in difficulty). The list contained nine words each exposed once, nine words each exposed three times, and nine words each exposed five times. Unlike the earlier study, list items were heterogeneous with respect to case format. For some list variants, 40 items were printed in uppercase and 41 in lowercase; for the other variants, 40 items were printed in lowercase and 41 in uppercase. Case placement in the overall sequence of items was randomized.

\section{Procedure}

Instructional variation followed the procedure of Kausler and Puckett (1980). Subjects performing the ancillary casemonitoring task were instructed to call out "same" or "different" for each item as it was exposed (i.e., "same" if the case matched that of the previous item and "different" if it did not match). Performance on this ancillary task was virtually perfect at each age level (maximum score $=80$ ), with mean scores of 79.04 and 79.21 for the young and elderly subjects, respectively $[t(46)<1]$.

Study list items were exposed for $5 \mathrm{sec}$ with an interitem interval of approximately $.5 \mathrm{sec}$. On the test trial, subjects received 18 index cards, each containing two words in leftright order (the more frequent word appearing equally often in each position). There were six types of pair combinations and three of each type: $0: 1,0: 3,0: 5,1: 3,1: 5$, and $3: 5$ (again, the numbers refer to study list frequencies). The test items were assigned randomly to the various pair types relevant to their actual exposures. For purposes of greater generalization, two different test lists were prepared, differing only with respect to the combinations of items. Testing was self-paced, with the cards being shuffled randomly for each subject. For each card, subjects called out the word they believed had occurred more frequently in the study list, with their choices recorded by the experimenter.

\section{RESULTS AND DISCUSSION}

An overall analysis of variance yielded a significant main effect for pair type $[F(5,440)=23.82, p<.001]$. The effect resulted from the overall greater difficulty of $3: 5$ and $0: 1$ pairs (mean correct $=2.24$ and 2.44 , respectively) than for the other pair types (mean correct $=2.81,2.96,2.54$, and 2.73 for $0: 3,0: 5,1: 3$, and $1: 5$ pairs, respectively). However, none of the interactions involving pair type approached significance $[\mathrm{Fs}(5,440)<$ 1.51]. Consequently, our further concern will be restricted to total frequency judgment scores on the combined pair types (maximum score $=18$ ). Means (and standard deviations in parentheses) for the young adults were 15.83 (1.50), 16.92 (1.06), 15.58 (1.92), and 17.00 (1.08) in the incidental/monitoring, incidental/ no-monitoring, intentional/monitoring, and intentional/ no-monitoring conditions, respectively. Comparable values for the elderly subjects were $14.42(2.15), 16.08$ (1.09), 13.92 (2.12), and 16.00 (1.73).

The analysis of variance for the composite scores revealed significant main effects for age $[F(1,88)=$ $12.33, \mathrm{p}<.001]$ and the presence/absence of the ancillary task $[\mathrm{F}(1,88)=19.92, \mathrm{p}<.001]$. The main effect for age reflected the higher mean score for young adult subjects (16.33) than for elderly subjects (15.10), and the main effect for ancillary task variation reflected the higher mean score for subjects performing without the task (16.50) than for subjects performing with the task (14.94). Both the main effect for instructions and all interaction effects involving age, ancillary task, and instructions fell far short of significance $[\operatorname{Fs}(1,88)<1]$.

The null effect for instructions replicates that found in earlier studies (e.g., Kausler \& Puckett, 1980). It adds to the evidence indicating the automaticity of encoding frequency of occurrence information. The absence of an interaction between age variation and instructional variation also replicates earlier results (Attig \& Hasher, 1980; Kausler \& Puckett, 1980). The implication is that frequency information is encoded automatically by elderly adults as well as by young adults. Additional support for the automaticity of encoding frequency information throughout the adult life span is provided 
by the absence of an interaction between age and the presence/absence of case monitoring. Although relative frequency judgments were less accurate overall in the ancillary task condition than in the standard condition, as expected, the reduction in accuracy was no greater for elderly subjects than for young adult subjects. Again, if frequency information is encoded less proficiently by elderly adults than by young adults, then the case-monitoring activity should have decreased performance proficiency to a greater extent for elderly subjects than for young adult subjects.

On the other hand, the significant main effect for age variation conflicts with the null effect reported earlier by both Attig and Hasher (1980) and Kausler and Puckett (1980). The present age difference resulted from the superior performance of the young adult subjects, rather than from the inferior performance of the elderly subjects. For example, the present young subjects had a mean score of 16.92 under incidental instructions and the absence of the ancillary task, whereas the young subjects in Kausler and Puckett's study had a mean score of 15.75 under identical conditions. In contrast, the mean scores of the present elderly subjects and those of Kausler and Puckett were nearly identical, 16.08 and 15.88 , respectively, under the same combination of conditions.

The primary difference between the samples of young adults in the two studies appears to be in their levels of fluid intelligence as assessed by the Figural Relations Test. The mean score was 10.52 for the present sample and 8.94 for Kausler and Puckett's (1980) sample. This difference assumes importance given the significant positive correlation between fluid intelligence and frequency judgment scores for young adult subjects, both in the present study $[\mathrm{r}(46)=.32, \mathrm{p}<.05]$ and in the earlier study $[\mathrm{r}=.60]$. Horn (1978) views fluid intelligence as an age-sensitive ability that influences a wide range of memory activities, activities that presumably are cognitively effortful. Consequently, memory proficiency is expected to covary positively with level of fluid intelligence. The critical question, of course, concerns the locus of a cognitively effortful activity on the frequency judgment task, an activity that might be expected to covary positively in proficiency with level of fluid intelligence.

The likely candidate for an effortful process is that of retrieval of frequency information. Young adults who are superior in fluid intelligence may excel in the retrieval of frequency information, relative to young adults of less superior ability, even when the retrieval requirements are minimized, as they are for relative frequency judgment tests. Frequency judgment scores for these superior young adults would therefore exceed those of elderly adults despite the comparability of the age groups in the encoding of frequency information. The magnitude of the age deficit should increase, in turn, as the amount of retrieval effort increases, as it does when subjects receive an absolute frequency judgment task. Consequently, age deficits in frequency judgment accuracy should be found more consistently with the absolute judgment task than with the relative judgment task, as appears to be the case. The present results suggest that an age deficit emerges on relative frequency judgment tests only when the young adults being sampled score especially high in fluid intelligence. This contingency seems less necessary in order for an age deficit to emerge on an absolute frequency judgment task. The relationship postulated here between fluid intelligence and the retrieval component of the relative frequency judgment task is, of course, derived only from correlational evidence. Corroboration of this relationship by means of other kinds of evidence is an important objective for future research.

\section{REFERENCES}

ATtig, M., \& Hasher, L. The processing of frequency of occurrence information. Journal of Gerontology, 1980, 35, 66-69.

Craik, F. I. M. Age differences in human memory. In J. E. Birren \& K. W. Schaie (Eds.), Handbook of the psychology of aging. New York: Van Nostrand Reinhold, 1977.

HAsher, L., \& ZaCKs, R. T. Automatic and effortful processes in memory. Journal of Experimental Psychology: General, 1979, 108, 356-388.

Hintzman, D. L., \& Block, R. A. Repetition and memory: Evidence for a multiple-trace hypothesis. Journal of Experimental Psychology, 1971, 88, 297-306.

HoRN, J. L. Human ability systems. In P. B. Baltes (Ed.), Lifespan development and behavior (Vol. 1). New York: Academic Press, 1978.

Kausler, D. H., \& PucketT, J. M. Frequency judgments and correlated cognitive abilities in young and elderly adults. Journal of Gerontology, 1980, 35, 376-382.

WArRen, L. R., \& Mitchell, S. A. Age differences in judging the frequency of events. Developmental Psychology, 1980, 16, 116-120.

\section{NOTE}

1. We are grateful to John L. Horn for his permission to use the Gf-Gc sampler in the present study and to the various granting agencies that have supported the development of the sampler.

(Received for publication August 24, 1981.) 